The Be Phenomenon in Early-Type Stars, IAU Colloquium 175

ASP Conference Series, Vol. 214, 2000

M. A. Smith, H. F. Henrichs, and J. Fabregat, eds.

\title{
Interferometry and Stellar Magnetism
}

\author{
O. Chesneau, K. Rousselet-Perraut and F. Vakili
}

Observatoire de la Côte d'Azur, UMR 6528, 06460 St Vallier de Thiey

Observatoire de Grenoble, UMR 5571, BP 53, 38041 Grenoble cedex 9

\begin{abstract}
The classical detection of magnetic fields in Be stars remains a challenge due to the sensitivity threshold and geometrical cancelation of the field effects. We propose to study the Zeeman effect using SpectroPolarimetric INterferometry (SPIN) which consists of the simultaneous use of polarimetry and very high angular resolution provided by long baseline interferometers. As monitoring of the instrumental polarisation is mandatory in order to calibrate interferometric observations in any case, the polarised signal is a natural by-product of interferometers. This method will be tested on the GI2T interferometer thanks to its high spectral resolution and its polarimetric capabilities.
\end{abstract}

\section{Introduction}

New developments in optical long-baseline interferometry to study the "disks" of Be stars (Stee, these proceedings) are of interest to Be star community. Using spectral Differential Interferomety (DI) we are now able to monitor variable structures of Be disks over long time scale (years) with milliarcsecond spatial resolution (Vakili et al. 1998; Bério et al. 1999). During this conference K. Bjorkman also showed that spectropolarimetry is a powerful tool to constrain the disk parameters, although it suffers from a lack of spatial resolution. It is therefore interesting to combine interferometry and polarimetry to detect local polarisation using Spectro-Polarimetric INterferometry (SPIN). Up to now few attempts have been reported (Vakili 1981), particularly on $\gamma$ Cas (Rousselet-Perraut et al. 1997). The efficiency of optical interferometers has improved dramatically in recent years, and with foreseen instruments such projects become more realistic. Here we focus on the huge potential of SPIN in detecting local and/or faint magnetic fields as proposed by Vakili (1984). We first describe the interferometric technical method to obtain polarimetric observables. Then we show preliminary results on the consequences of a structured dipolar or quadrupolar magnetic field on polarisation intensity maps, and the corresponding interferometric observables. Finally, we discuss the application of this technique for the detection of magnetic fields of Be stars. 

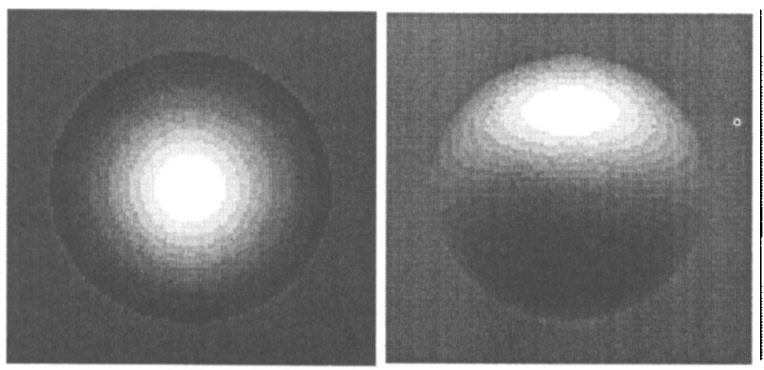

Figure 1. Stokes V parameter maps for a dipole. In the pole-on view (left), the polarised light mimics a change in limb-darkening. In the edge-on view (right), the map photocenter is shifted.

\section{Differential Interferometry}

DI uses high angular resolution capabilities of interferometry in dispersed light in order to compare the spatial properties of an object at different wavelengths. This technique offers obvious advantages (Vakili 1994). Among them, differential analysis increases the sensitivity and allows one to retrieve relative phase information across the spectrum. Thus, two observables become available: the classical "visibility-modulus" Vm and the relative phase $\phi_{r}$ related to the emission photocenter. Using continuum fringes as phase reference, one can for instance follow the light photocenter across a spectral line. In the particular case of Be stars, departures from symmetry observed in spatially resolved emission lines (due to disk inhomogeneities) are referred to the unresolved continuum. In the case of circularly polarised light, two interference patterns are obtained with opposite states, and in each, the continuum can be considered as an unpolarised reference. The Zeeman effect splits magnetically sensitive photospheric lines, and induces circular polarisation on sigma components. This polarisation is the integrated contribution of the intensity map modulated by the polarised pattern (Stoke V parameter). The longitudinal field intensity is not constant over all the disk, and rotational Doppler effect spreads the line. As a consequence, the polarisation is diluted on the line and the polarised intensity maps depend highly on the location and the width of the spectral channel under study. By following the visibility and the phase through the line at different epochs, we obtain information on both the geometry and intensity of the field.

\section{SPIN observations}

Figure 1 displays the Stokes $\mathrm{V}$ parameter computed on the stellar surface in a small spectral channel localised in a sigma component of a Zeeman triplet. This figure depicts the relative intensity of the longitudinal magnetic field for a dipolar configuration. Edge-on and pole-on configurations induce quite different interferometric signatures. A pole-on configuration (left) is centro-symmetric so that no phase effect occurs. But the star appears smaller/larger, thus changing its visibility Vm (the polarisation effect is maximum). Using increasing base- 


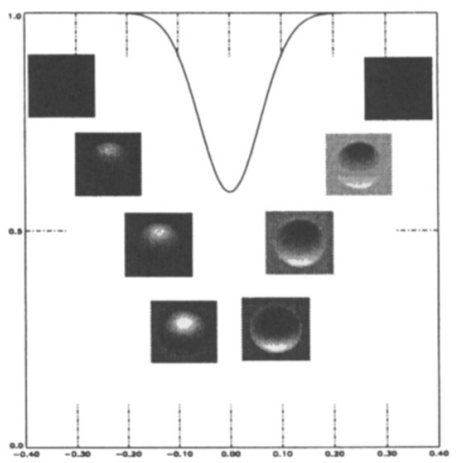

Figure 2. Zeeman effect on quasi-monochromatic intensity maps: $i=60^{\circ}, H_{\mathrm{p}}=4000 \mathrm{G}, \mathrm{R}=60000$. Noticeable effects are detected only on the four centered channels.

lines, and by comparing polarised and unpolarised signals, we can disentangle limb-darkening and magnetic effects.

Note that the edge-on configuration is undetectable by classical techniques due to the cancelation of opposite magnetic poles. Visibility effects on Vm are minimum, since the projected field is minimum, although they are not null. In this case (right), the polarised pattern is antisymmetric, and a large photocenter shift effect occurs. As the polarisation is inverted in opposite sigma components, the corresponding phase shifts $\Delta \phi_{r}$ become opposite too. This effect is similar to the familiar $S$ curve of spectropolarimetrists.

The same considerations apply with quadrupolar fields, with some kind of signal attenuation. In each case, many baseline orientations are necessary to constrain the inclination $i$, the obliquity $\beta$, the polar field $H_{\mathrm{p}}$ and the orientation of the rotation axis on the sky. We emphasize that the SPIN method is sensitive to the field strength and its spatial configuration.

\section{Small field detection: a typical example}

We consider a slowly rotating star with an apparent diameter of 0.7 mas, observed at a spectral resolution of $R=30000$. The considered magnetic field is dipolar or quadrupolar. Note that the visibility signal $\mathrm{Vm}$ of an interferometer depends on the square root of the collected flux whilst its phase $\phi_{r}$ goes linearly with this flux. If the visibility alone $\mathrm{Vm}$ is used, an angularly resolved magnetic star demands very large collecting primary mirrors for its interferometric observation (e.g. VLTI, Keck interferometer). For instance with a $200 \mathrm{~m}$ baseline a 0.7 mas star is almost completely resolved, i.e. the fringe visibility is quasi-null. Instead, using the phase signal makes possible to detect an edge-on dipolar magnetic field as low as $100 \mathrm{G}$, with a $1^{\circ}$ phase accuracy at a $200 \mathrm{~m}$ baseline even with $1 \mathrm{~m}$ class interferometers. This should be moderated by the fact that the Zeeman effect necessarily requires very narrow spectral channels $(<0.2 \AA)$, hence long exposures exceeding 30 minutes to attain the required SNR. For quadrupoles, Vm effects are no longer useful whereas the phase $\phi_{\tau}$ 
effect remains noticeable : a $400 \mathrm{G}$ edge-on quadrupole is still detectable. Using $R=60000$, the limits of detectability are significantly improved with the same sensitivity: $100 \mathrm{G}$ at $100 \mathrm{~m}$ baselines and $200 \mathrm{G}$ at $50 \mathrm{~m}$. Indeed, magnetic field detection highly depends on the spatial configuration of the field. Contrary to spectropolarimetry, edge-on field configurations are more favorable to detect than pole-on ones where no phase effect can occur. Our group is currently quantifying these simulations with the GI2T interferometer.

\section{Be star magnetic fields}

Some Be stars are bright and relatively close, i.e. resolvable. They may have fossil magnetic fields, and preferentially quadrupolar (see the Dudorov contribution). The magnetic field should be studied in photospheric lines, where the field is supposed to be stronger. For the closest Be stars, photospheric angular diameters range between 0.75 mas and 0.3 mas; baselines larger than $100 \mathrm{~m}$ are thus required. As a first step, our computations are restricted to the Zeeman effect neglecting the large rotational Doppler effect presented by these stars. The results described above are thus only illustrative and concern pole-on Be stars like $\eta$ Tau. SPIN is devoted to two main goals: detecting and studying magnetic fields. For Be stars, the detection of global/local magnetic fields is clearly a priority. In particular, SPIN should be highly sensitive to localized spots in stars such as $\gamma$ Cas (Smith and Robinson, these proceedings). A magnetic spot on the unpolarised surface of the star appears in a polarised phase diagram as a localised S curve which moves across the line. In that sense, Doppler Imaging techniques can apply to SPIN observations, allowing one to monitor of the magnetic activity. Moreover, some Be star are among the few stars where the exact orientation of the rotation axis is known thanks to polarimetry and previous interferometric observations (Quirrenbach 1997), which decreases the free parameters. No doubt that this technique -as for all polarisation techniques- needs a huge amount of photons, along with combined adaptive optics and interferometry. Observational tests will be done using the polarimetric and spectral facilities of GI2T (Thureau, these proceedings). With $R=30000$ and $60 \mathrm{~m}$ baseline, polar fields weaker than $4000 \mathrm{G}$ are clearly undetectable. Well known highly magnetized Ap stars such as $\beta \mathrm{CrB}$ will therefore be considered first.

\section{References}

Berio, P., Stee, P. Vakili, F. et al. 1999, A\&A 3350, 517

Mourard, D., et al. 1998, SPIE 90, 473

Rousselet-Perraut, K., Vakili, F. 1997, A\&AS 123, 173

Quirrenbach, A., et al. 1997, ApJ 479, 477

Vakili, F. 1981, A\&A 101, 352

Vakili, F. 1984, SPIE 323, 183

Vakili, F., Mourard, D., Stee, P. 1994, IAU Symposium 162 (Eds. L.A. Balona, H.F. Henrichs, M. Le Contel), p. 435

Vakili, F., Mourard, D. et al. 1998, A\&A 323, 183 